\title{
Peat as an alternative material for use in ammonia sensors
}

\author{
N.C.Ribeiro ${ }^{1}$, N.S.Pereira ${ }^{2}$, H.H. Guimarães ${ }^{3}$, G.L.Sandri', and A.M.Ceschin ${ }^{3}$ \\ Instituto Federal de Mato Grosso - Campus Rondonópolis - MT - Brazil \\ ${ }^{2}$ Insituto de Química da Universidade de Brasília - Campus Darcy Ribeiro - DF - Brazil \\ ${ }^{3}$ Departamento de Engenharia Elétrica da Universidade de Brasília - Campus Darcy Ribeiro - DF- \\ Brazil-CEP:70904-970 artemis@ene.unb.br
}

\begin{abstract}
This work consists in a study of an alternative material to be use for the development of ammonia sensors. The humin is extracted from peat with centrifugation of $\mathrm{KOH}$ or $\mathrm{NaOH}$ solutions. The humin consists basically of carbon. The powder of humin is a porous material, similar to zeolita, and it is partly solubilized in water. The solubilized humin is deposited on a flexible polyester substrate and dried in an oven at $80^{\circ} \mathrm{C}$ for 20 minutes. The samples' sensitivity to ammonia vapor was measured using the standard two-temperature method.
\end{abstract}

Key words: humin, porous, ammonia, sensors.

\section{Introduction}

Measurement of ammonia vapor is required in numerous applications including the chemical and beverages industries and cooling systems [1].

Recent advances in ammonia-sensitive materials explored the use of zeolita [2] and conjugated polymers.[3]. Based on the changes in electrical or optical properties, different ammonia sensors have been proposed. The sensors based on electrical properties, such as impedance, resistance or capacitance, are best suited for modern automatic control systems. Electrical detection is the most commonly used sensing method, which is based on the change of sensor's resistance or capacitance when exposed to ammonia vapor or gases [4].From their basic principles, they are classified into two categories: 1) based on the change in the electrical properties of the material due to the absorption of ammonia vapor; 2 ) based on the gravimetric change in the material such as the quartz crystal oscillator. The first category is divided into two types, the electric resistance type and the capacitor type. Hydrophilic materials are used for resistance-type humidity, ammonia vapor or gases sensors while the hydrophobic materials are preferred for capacitance type sensors.

This paper reports an evaluation of a different organic material (humin) as a simple ammonia sensor for a range of ammonia vapor pressures. The humin is hydrophilic, so the sensors that will be studied here are based on the variation of electrical resistance. In this work, a plastic substrate (polyester sheet) is used to guarantee the flexibility of the sensor.

\section{Materials and sample preparation}

The powder of peat was obtained from 200 $\mathrm{g}$ of an in natura peat sample, maintained in an oven for 4 hours for drying. Then, the peat sample was finely grounded in a ball mill, until a very thin powder, with grains smaller than 20 $\mathrm{mm}$, was obtained. The samples to be analyzed are those obtained with the first, second and third extraction, using $\mathrm{KOH}$ and $\mathrm{NaOH}$ (i.e., a total of six samples). The humin contain nanoporous, whose surface area was measured by ITS and pore volume was measured using a BET isotherm (Brunauer, Emmett, Teller), with liquid and gaseous nitrogen. The diammeter of the nanoporous ranged from 20 to $30 \mathrm{~nm}$ in average. The humin was deposited over the $1.5 \mathrm{~cm} \times 4.0 \mathrm{~cm}$ polyester substrate, by casting. After those films were deposited on the substrate, the samples were placed in an oven at $40^{\circ} \mathrm{C}$ for approximately 20 minutes.

\section{Experimental setup}

The samples' sensitivity to ammonia vapor was measured using the standard twotemperature method. A closed system, similar to that of Ansari et al. [5], was used for testing the ammonia response of the films. It consists of a close flask, with two necks for inserting thermometers (in the range -10 to $200^{\circ} \mathrm{C}$ with $1.0^{\circ} \mathrm{C}$ accuracy), $100 \mathrm{ml}$ filled with $30 \%$ ammonia solution (total volume $500 \mathrm{ml}$ ). The flask is kept in a container filled with ice to the same level of ammonia solution in the flask. The temperature of the system is adjusted by mixing ice and water as required. Thus, the water inside the flask can be kept at the required temperature $\left(T_{2}\right)$. The sample is mounted inside the flask at a height of $3 \mathrm{~cm}$ 
from the level of ammonia solution and the temperature of the sample $\left(T_{1}\right)$ is measured with a thermometer placed at the height of the sample under study. The temperature of the sample changes by $2-42^{\circ} \mathrm{C}$ during the experiment.

The relative vapor pressure $(\mathrm{RH})$ inside the chamber is calculated by the equation (1). The $\mathrm{RH}$ of the vapor in the system is given by equation (1):

$\% R H=E_{w}\left(T_{2}\right) / E_{w}\left(T_{1}\right) \times 100$

Where, $E_{w}\left(T_{2}\right)$ is the saturated vapor pressure at the temperature of the ammonia solutionbath $\left(T_{2}\right)$ and $E_{w}\left(T_{1}\right)$ is the saturated vapor pressure at the temperature of the sample element $\left(T_{1}\right)$. The values of the saturated vapor pressure were obtained from reference [6]. The ranges of $\mathrm{RH}$ values are obtained by adjusting the temperature of the ammonia solution inside the flask, with ice and water mixture from room temperature to $0^{\circ} \mathrm{C}$. The change in relative vapor pressure is related to the change in the resistance of the sample. The electrical characterization of the samples described above, while the relative vapor pressure varied inside the chamber, was performed with the programmable measurement system Keitley 2400. A direct bias was applied with a voltage swept from $-5 \mathrm{~V}$ to $5 \mathrm{~V}$. Both voltage and current were registered into a table to calculate the resistance.

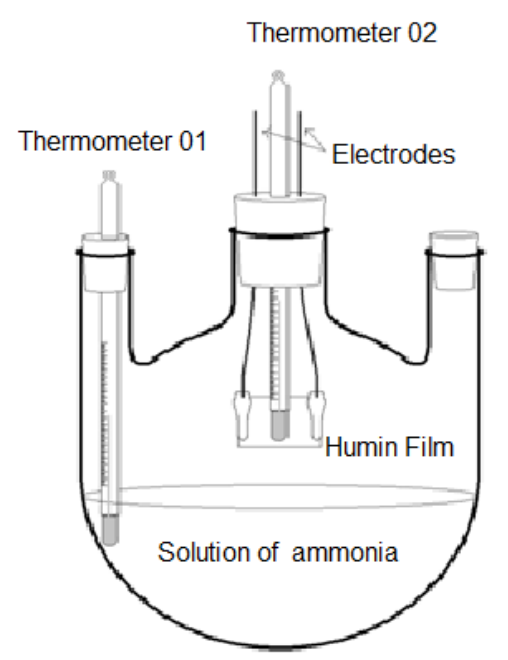

Fig. 1. Experimental setup for the measurement of vapor pressure of ammonia.

\section{Results and discussion}

The ammonia has a state of equilibrium that is established according to the system temperature. The reaction equilibrium is governed as follows: $\mathrm{NH}_{4}^{+}{ }_{(\mathrm{aq})}+\mathrm{OH}_{(\mathrm{aq})}^{-} \leftrightarrow \mathrm{NH}_{3}$ (g) $+\mathrm{H}_{2} \mathrm{O}(\mathrm{I})$. At temperatures above $30^{\circ} \mathrm{C}$, it is possible to clearly observe the phenomenon of surface adsorption. The films deposited by casting are thick, thus a saturation of the ammonia vapor adsorption was observed in all samples, because this is a superficial effect. The repeatability of ammonia sensing using humin was found to be reasonable for all Figure 2 shows the $1 \times \mathrm{V}$ curve for the third extraction of humin with $\mathrm{KOH}$, repeated three times. Between each measurement, the sample was placed in an oven at $40^{\circ} \mathrm{C}$ for 10 minutes for ammonia desadsorbing. The curves are not exactly equal. This could be due to imprecision associated with the twotemperature method. Also, if the response time is not fast enough, this can induce measurement errors. Figure 3 shows a plot between relative ammonia vapor and resistance of the sensor, for the six samples. As the vapor pressure of ammonia increases, the resistance of the sample decreases.

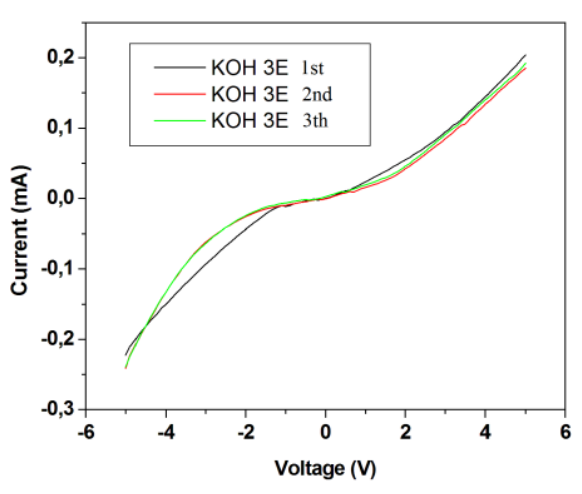

Fig.2: Repeatability result for third extraction of $\mathrm{KOH}$ sample

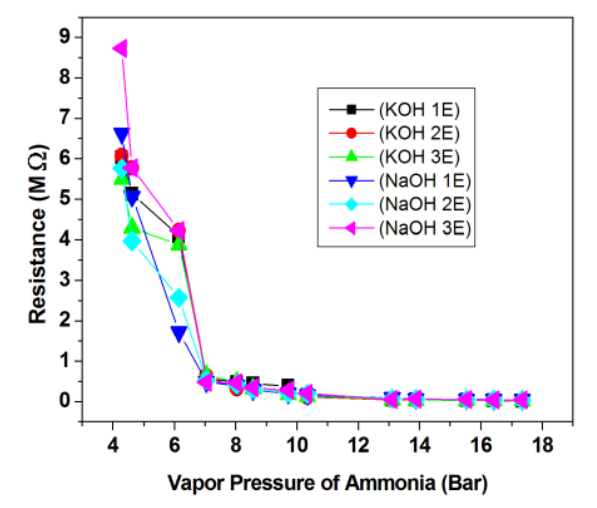

Fig.3: Variation of resistance with respect to ammonia vapor for the six samples of humin from peat.

\section{Conclusion}

In this work, a new material (humin) deposited on a flexible polyester substrate was used to fabricate an ammonia sensor. The humin of peat was effective as an ammonia sensor, as the samples' resistance decreased with the ammonia vapor uptake. 


\section{References}

[1] Tamaru, K. Em The History of the Development of Ammonia Synthesis in Catalytic Ammonia Synthesis: Fundamentals and Practice, Jenning, J. R., ed., Plenum Press: New York, 1991

[2] M.B.Filho,H.G.Riter,M.G.daSilva,F.J.Luna, C.G.Werneck,I.Rech,J.C.Polidoro,M.B.M.M onte,F.S.Barros,A.Mikloa

[3] and H.Vargas, Sensors and Actuators B:Chemical 158,241-245 (2011)

[4] Harrison, I.M., Meadows, J.; Robb, I.D., Willians, P. "Competitive Adsorption of Polymers and Surfactants at the Solid/Liquid Interface" J. Chem. Soc. Faraday Trans, 91(21), 3919-3923 (1995)

[5] L.da Silva, F. E. da Silva, C.V.Franco,R.B.Nuernberg,T. Gomes, R.Miranda and M.M.da Silva Paula,Mater.Sci.and Eng. C, 599 (2009)

[6] S.G.Ansari,.A.Ansari,M.R.Kadam,R.N.Kare kar, R.C.Aiyer, Sens. Actuators B, 21, 159 (1994)

[7] G. D. Farquhar, P. M. Firth, R. Wetselaar, and $B$. Weir - On the Gaseous Exchange of ammonia between Leaves and the Environment: Determination of the Ammonia Compensation Point 1980.

[8] Smil, V.; Enriching the Earth, MIT Press: Cambridge, Massachusets, 2000. 